\title{
Reflectance confocal microscopy characteristics of oral lichen planus: An analysis of 47 cases in a Chinese cohort
}

\author{
HAO PENG ${ }^{1,2}$, YUFENG WANG ${ }^{3}$, LINGYUE SHEN ${ }^{1,2}$ and GUOYU ZHOU ${ }^{1,2}$ \\ ${ }^{1}$ Department of Oral and Maxillofacial-Head Neck Oncology, Shanghai Ninth People's Hospital, College of Stomatology, \\ Shanghai Jiao Tong University School of Medicine, National Clinical Research Center for Oral Diseases, \\ Shanghai Key Laboratory of Stomatology and Shanghai Research Institute of Stomatology; \\ ${ }^{2}$ Department of Laser and Aesthetic Medicine, Ninth People's Hospital, Shanghai Jiao Tong University School of Medicine; \\ ${ }^{3}$ Department of Oral Medicine, Shanghai Ninth People's Hospital, College of Stomatology, \\ Shanghai Jiao Tong University School of Medicine, National Clinical Research Center for Oral Diseases, \\ Shanghai Key Laboratory of Stomatology and Shanghai Research Institute of Stomatology, \\ Shanghai 200011, P.R. China
}

Received December 23, 2019; Accepted June 10, 2020

DOI: $10.3892 / \mathrm{etm} .2020 .9134$

\begin{abstract}
Reflectance confocal microscopy (RCM) is a non-invasive tool that provides real-time microscopic images and relatively high-resolution tissue images. This technique provides a link between clinical examination and histopathology. RCM has been used to detect skin diseases and has also recently been applied to diseases of the oral mucosa. The present study aimed to explore the features of oral lichen planus (OLP) using RCM. A total of 47 patients with OLP exhibiting a reticular pattern, were included in the present study. The lesion sites and healthy adjacent sites were examined using in vivo RCM, with the lesion being histopathologically confirmed after RCM examination. The confocal images were reviewed, and the features were described. Sensitivity and specificity analysis of the RCM features was also performed. $\mathrm{RCM}$ examination presented parakeratosis, acanthosis and
\end{abstract}

Correspondence to: Dr Guoyu Zhou, Department of Oral and Maxillofacial-Head Neck Oncology, Shanghai Ninth People's Hospital, College of Stomatology, Shanghai Jiao Tong University School of Medicine, National Clinical Research Center for Oral Diseases, Shanghai Key Laboratory of Stomatology and Shanghai Research Institute of Stomatology, 639 Zhizaoju Road, Shanghai 200011, P.R. China

E-mail: guoyu_zhou@126.com

Dr Yufeng Wang, Department of Oral Medicine, Shanghai Ninth People's Hospital, College of Stomatology, Shanghai Jiao Tong University School of Medicine, National Clinical Research Center for Oral Diseases, Shanghai Key Laboratory of Stomatology and Shanghai Research Institute of Stomatology, 639 Zhizaoju Road, Shanghai 200011, P.R. China

E-mail: dr_wangyufeng@126.com

Key words: reflectance confocal microscopy, oral lichen planus, noninvasive diagnosis connective tissue papillae disappearance, with the presence of large melanocytes and roundish inflammatory cell infiltration, as well as dilated vessels in the lesion tissue. The sensitivity and specificity of OLP for dorsal tongue lesions were not as satisfactory as those on other sites. The results implied that RCM may be a promising technique to detect OLP non-invasively in vivo.

\section{Introduction}

Lichen planus is a chronic inflammatory disease that effects the skin and oral mucosa (1). It affects $\sim 1-2 \%$ of people over 40 years worldwide (2). As with oral mucosa, buccal mucosa, the tongue and gingiva are the main tissues affected (3). Oral lichen planus (OLP) is commonly affected by bilateral lesions and presents clinically in multiple forms, such as reticular, papular, plaque-like, erosive, atrophic and bullous (4). OLP is a potentially malignant disorder of the oral mucosa (5), indicating that it may undergo malignant transformation (6). Repeated biopsies are required for suspected lesions. Consequently, a non-invasive tool for assisting in the follow-up of ongoing diseases is urgently required within clinics. A number of non-invasive techniques have been developed, such as toluidine blue and Lugol's iodine vital staining, autofluorescence, chemiluminescence and brush biopsy (7). The use of these adjuncts is useful and helps to increase clinical diagnostic sensitivity and specificity of OLP compared to clinical examination alone, even if not markedly (7).

Reflectance confocal microscopy (RCM) is a novel in vivo infrared optical detection device that has been widely used in the examination of dermatological $(8,9)$ and oral mucosal diseases $(10,11)$ in recent years. RCM provides real-time horizontal frame images at the cellular level, which can reach the superficial dermis (12). The contrast of RCM images relies on the different refractive index of subcellular components (13). A number of studies assessing the application of RCM in OLP have been previously reported and RCM cellular and 
architectural findings have been described (14-17). However, to the best of our knowledge, relevant studies in a Chinese cohort have not been performed. The present study aimed to observe the RCM features of OLP in a Chinese population.

\section{Materials and methods}

Patients. A total of 47 patients (25 males and 22 females) who were clinically diagnosed with OLP, with a mean age of 49.4 years (range, 29-74), were enrolled at the Ninth People's Hospital, Shanghai Jiao Tong University, School of Medicine between April 2015 and June 2016 (Table I). The patients received an oral dose of fluconazole $50 \mathrm{mg}$ once a day and a $1 \%$ sodium bicarbonate mouth wash three times a day for two weeks. Inclusion criteria were patients with newly diagnosed OLP exhibiting a reticular pattern, which was clinically diagnosed by an oral medicine specialist and confirmed by subsequent histopathological examination according to modified World Health Organization (WHO) diagnostic criteria (18). Exclusion criteria were lesions with erosion and ulceration. All patients were informed about the purpose and procedure of the study and provided written informed consent. Ethical approval for this investigation was obtained from the Ethics Committee of Shanghai Ninth People's Hospital, Shanghai Jiao Tong University School of Medicine (approval no. 2014038).

In vivo reflectance confocal microscopy (RCM). A commercial RCM (Vivascope 3000; Lucid, Inc.) with a light source of a near-infrared wavelength $(830 \mathrm{~nm})$ stimulated by a diode laser at the variable power of $0-22 \mathrm{~mW}$ was used in the present study. The system included a $30 \mathrm{x}$ water immersion objective lens with a numerical aperture of 0.9. A tissue ring was attached to the lens after ultrasound gel was interposed between the lens and the coverglass. The images were displayed in 1,000x1,000-pixel horizontal sections. The lateral resolution could reach $0.5-1 \mu \mathrm{m}$. The Vivastack mode was taken to obtain sequential sections of vital tissue at intervals of $5 \mathrm{~mm}$ from the superficial layers to the submucosa. Patients were examined using RCM and the healthy contralateral sides of the lesion area were also examined using RCM as a control.

RCM features, including parakeratosis, acanthosis, liquefaction degeneration, inflammatory cell infiltration and dilated blood vessels were evaluated. In addition, RCM features were analyzed for their sensitivity and specificity for evaluating the accuracy of RCM. Sensitivity is presented as the percentage of OLP lesions with the RCM features and specificity is presented as the percentage of healthy areas without RCM features.

Biospy preparation. Each lesion underwent an incisional biopsy $(\sim 5 \times 5 \mathrm{~mm})$ after two weeks of treatment, using a scalpel blade under local anesthesia (2\% lidocaine with epinephrine) at the same lesion site that was used for the RCM analysis. Biopsy specimens were routinely fixed in $10 \%$ phosphate-buffered neutral formalin for $24 \mathrm{~h}$ at room temperature $\left(20-25^{\circ} \mathrm{C}\right)$, embedded in paraffin and sectioned vertically in the traditional manner. Subsequently, 5-mm thick sections were stained with hematoxylin for 5-10 min and eosin for 1-5 min at room temperature $\left(20-25^{\circ} \mathrm{C}\right)$. Two experienced pathologists performed histopathological evaluations using a light microscope (BX51; Olympus Corporation) with a digital
Table I. Patient demographics.

\begin{tabular}{lc}
\hline Demographic & Value \\
\hline Sex (no.) & \\
Male & 25 \\
Female & 22 \\
Mean age (years) & $49.4 \pm 13.3$ \\
Localization (no.) & \\
Lower lip (dysplasia) & $11(1)$ \\
Upper lip & 4 \\
Dorsal tongue (dysplasia) & $21(4)$ \\
Ventral surface of the tongue & 5 \\
Buccal mucosa (dysplasia) & $6(2)$ \\
\hline
\end{tabular}

Data are presented as the mean \pm standard deviation.

camera (DP71; Olympus Corporation) under x100, x200 and x400 magnification.

\section{Results}

Study population. The present study was comprised of 25 males and 22 females. A total of 47 lesions and 47 perilesional healthy areas were examined: 11 on the lower lip, 4 on the upper lip, 21 on the dorsal tongue, 5 on the ventral surface of the tongue, 6 on the buccal mucosa and 0 on the gingiva. A total of 7 patients presented with histopathological dysplasia. Table I presents the demographic characteristics of the patients that were enrolled.

RCM features. In the healthy tissue sites, keratinocytes were identified by their outlines and the rounded highly refractive nucleolus was observed within the cells of the thin superficial layer (Fig. 1A). At the stratum spinosum, smaller cells presented a honeycomb-like architecture (Fig. 1B). At the level of the epithelial-connective tissue junction, connective tissue papillae and blood flowing inside the vessels could be seen in vivo, especially on the lips (Fig. 1C). Connective tissue could also be observed in the dermis, although not very clearly (Fig. 1D). In the specialized lingual epithelium, filiform papillae exhibited elongated sickle-like structures and the taste buds consisted of large, spindle-shaped cells.

In OLP, at the superficial layers, hyperkeratosis could be detected (Fig. 2A). A greater number of layers were identified in the stratum spinosum in OLP lesions than in normal sites using the Vivastack mode, which meant that acanthosis could be observed (Fig. 2B). Keratinocytes presented larger at this layer. At the epithelial-connective tissue junction, the ring-like bright structures disappeared due to necrosis of the keratinocytes. Non-rimmed papillae were obscured by roundish, small inflammatory cells (Fig. 2C). Dark lumen structures with bright round cells located inside these structures were visible in the papillary dermis, which corresponded to dilated vessels with inflammatory cell infiltration (Fig. 2D). Bright large dentritic structures, which were interpreted to be melanophages, could be seen in the dermis. 

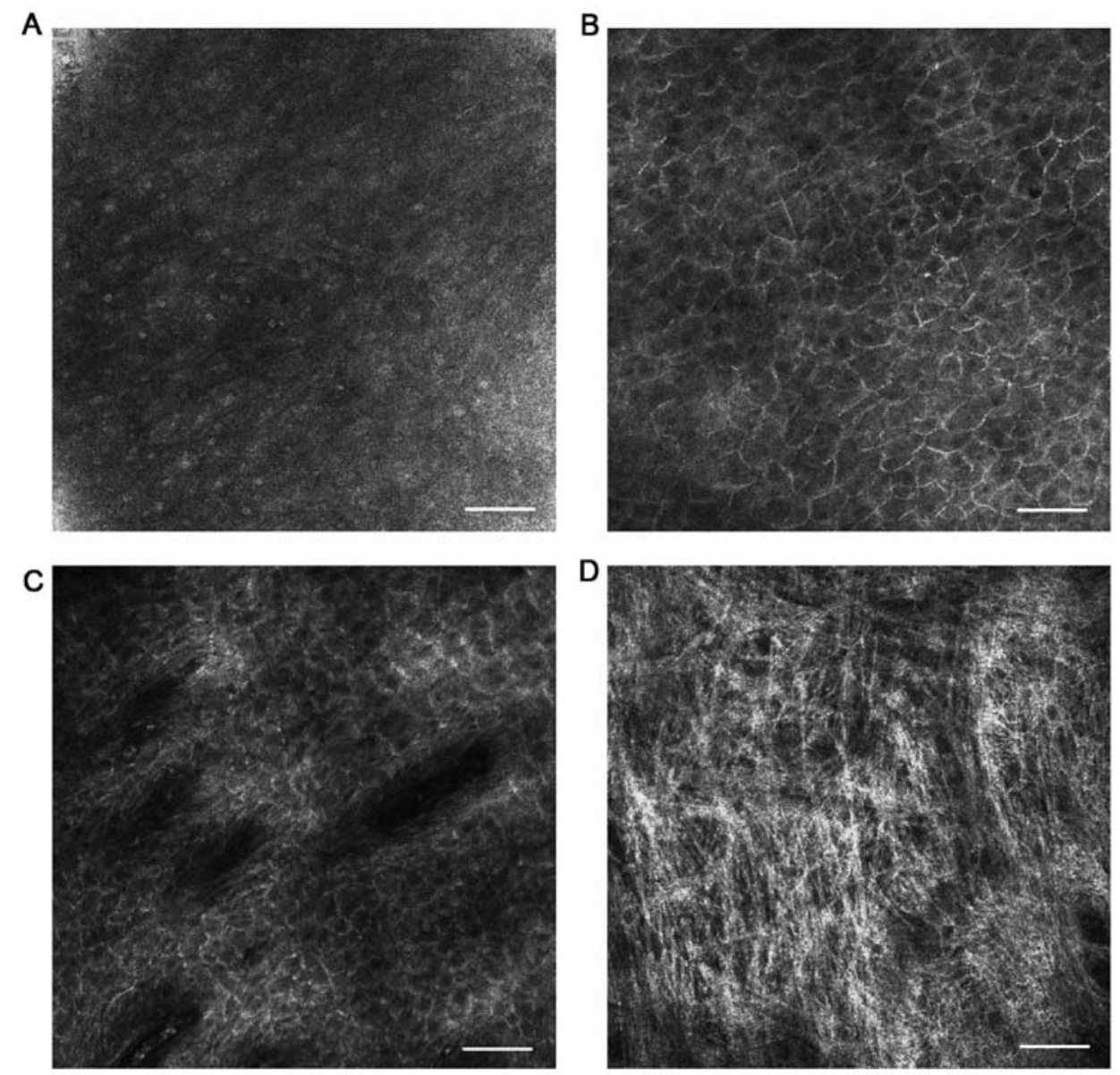

Figure 1. Reflectance confocal microscopy horizontal frame section of normal labial mucosa. Keratinocytes were regularly arranged at (A) the superficial layer and (B) and stratum spinosum. (C) Keratinocytes and connective tissue papillae were clearly observed at the epithelial-connective tissue junction. (D) Collagen fibers were identified at the lamina propria. Scale bar, $100 \mu \mathrm{m}$.

Statistical analysis. Sensitivity and specificity were calculated. The sensitivity and specificity of inflammatory cells were 0.70 and 0.87 , respectively. The values of non-rimmed papillae were 0.47 and 1.00 , respectively. The sensitivity of non-rimmed papillae of lesions on the dorsal tongue was reduced to 0.29 (Table II).

Epithelial dysplasia. In a total of 7 cases, histopathological examination revealed various degrees of dysplasia. Corresponding features were also identified using RCM imaging, such as keratinocytes which varied in size, disarrangement at the stratum spinosum (Fig. 3B) and multi-nucleolated cells (Fig. 3A) being occasionally detected. Intercellular dark spaces and neoangiogenesis were also observed (Fig. 3C).

\section{Discussion}

OLP is a chronic inflammatory condition that is caused by autoimmune disorders (19). OLP may manifest in various ways, such as in a reticular-plaque pattern or as erosive lesions and plaques (20). The plaque type of OLP can resemble leukoplakia by visual inspection and palpation (21). Furthermore, OLP is considered to be a disease that results in a significantly increased risk of developing cancer (22). As such, it is essential for patients with OLP to visit a doctor regularly. A rapid noninvasive tool would be beneficial for clinical diagnostic evaluations during patient follow-ups. RCM has been used recently for oral mucosal diseases.

A number of studies have been published investigating the applications for RCM in OLP. Contaldo et al (14) reported parakeratosis, hypergranulosis, acanthosis, necrotic keratinocytes, disrupted connective tissue papillae and inflammatory cell infiltration in RCM images of OLP tissue. Alessi et al (16) corroborated these findings and described melanophages as bright, large dendritic cells in five cases. In the present study, hyperkeratosis, parakeratosis and acanthosis were observed. At the level of the epithelial-connective tissue junction, the disappearance of bright ring-like structures indicated the destruction of the basal layer (14), which corresponded with the observed histopathological characteristics (23). Papillary rims may be obscured by the presence of inflammatory cells, which present as brightly refractive, roundish structures, but unlike in histopathology it was not possible to further distinguish the inflammatory cells (24). Dilated vessels and melanophages were also identified in the upper dermis.

Maturational disorders may occur in OLP due to its chronic inflammatory condition (25). Contaldo et al (14) briefly described multi-nucleolated cells and structures that resembled keratin pearls, which were identified in two lesions upon RCM examination. The WHO has deemed OLP as a premalignant disease (25). A previous study also demonstrated that the reticular type of OLP had the highest rate of malignant 

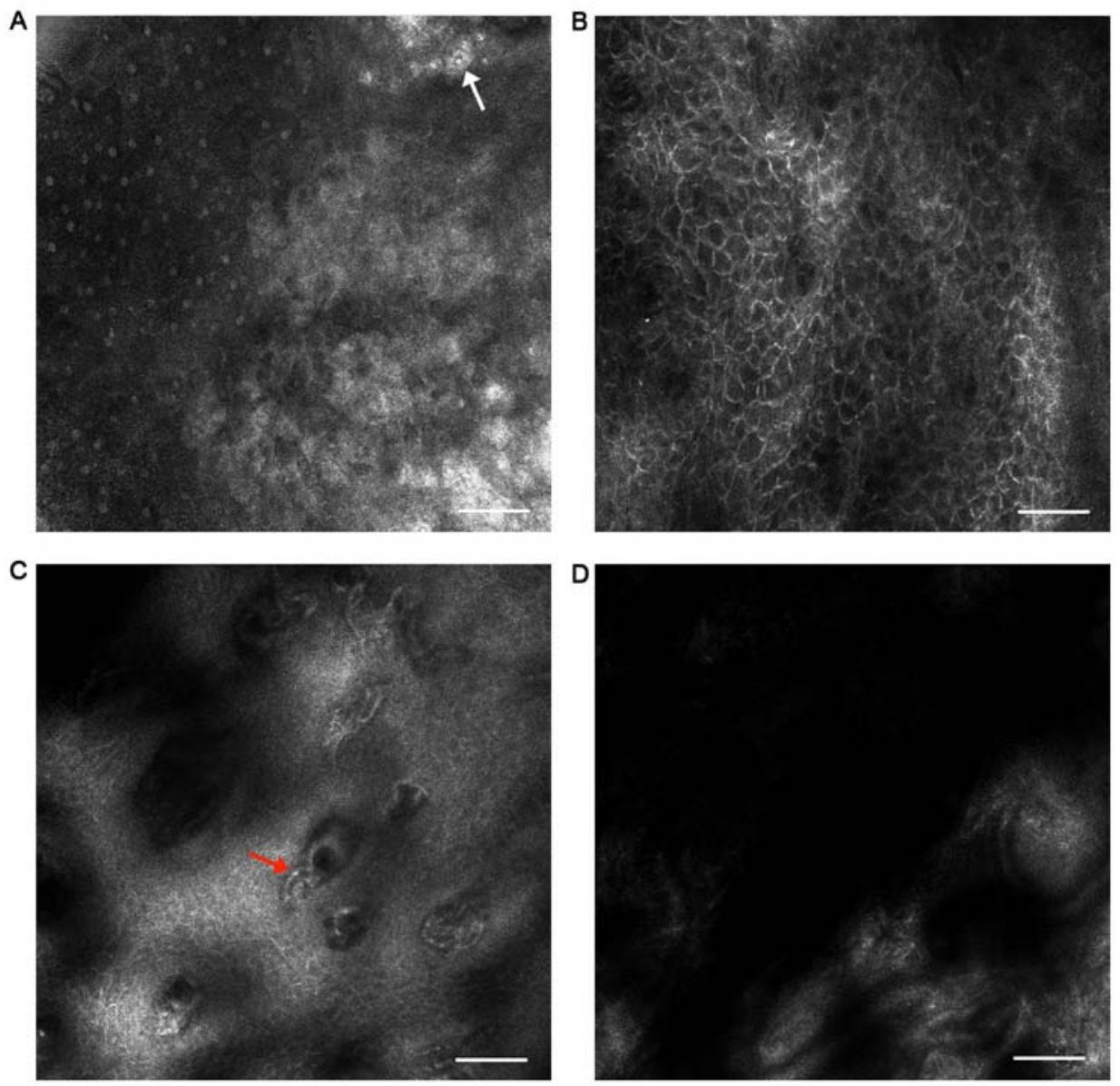

Figure 2. Reflectance confocal microscopy horizontal frame sections of oral lichen planus of the cheek. (A) Hyperkeratosis was exhibited with bright structures and parakeratosis (white arrow) at the superficial layer. (B) Keratinocytes were arranged regularly at the stratum spinosum. (C) At the level of the epithelialconnective tissue junction, non-rimed connective tissue papillae were observed. Small roundish inflammatory cells infiltrated through the dilated vessels (red arrow). (D) Below the basal layer, connective tissue papillae appeared disrupted and dilated vessels could be detected by a black lumen. Scale bar, $100 \mu \mathrm{m}$.
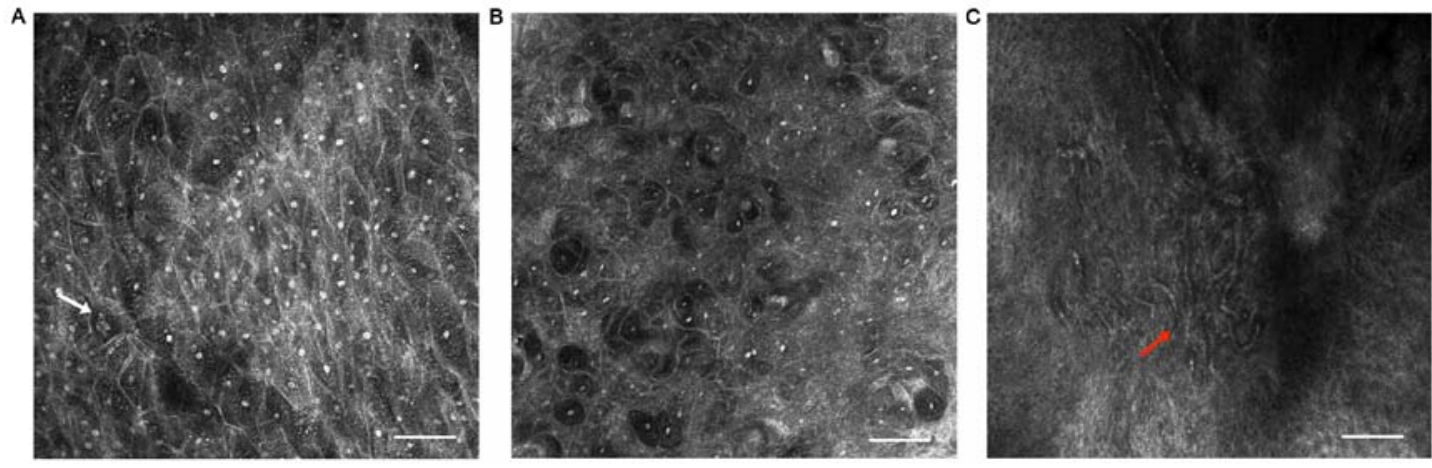

Figure 3. Reflectance confocal microscopy horizontal frame sections of features of dysplasia. (A) At the stratum spinosum, keratinocytes seemed larger than those in normal mucosa. Multinucleolated keratinocytes could also be detected (white arrow). (B) At the stratum spinosum, keratinocytes differed in size and shape, and were arranged irregularly. (C) Neoangiogenesis could be observed below the basal layer. Scale bar, $100 \mu \mathrm{m}$.

transformation (26). In the present study, a total of 7 biopsy samples were revealed to exhibit various degrees of epithelial dysplasia. Cellular disarrangement, pleomorphism, dispersed nuclei and large intercellular spaces were also identified. In 2019, Contaldo et al (15) reported that the most characteristic RCM criteria for malignancies were cytological and architectural disarray in well-differentiated oral squamous cell carcinoma, as well as cellular pleomorphism, increased nuclear-cytoplasmic ratios and multi-nucleolated keratinocytes in moderately/poorly differentiated oral squamous cell carcinoma. These findings were similar to those in the present study, which indicated that these features may suggest underlying cancerous changes in OLP lesions. However, only few features of dysplasia were detected by RCM in the present study. Further work is required to assess the use of RCM in the diagnosis of precancerous lesions.

In the present study, the sensitivity and specificity of the RCM features on the dorsal tongue were lower compared with 
other sites, such as lips and buccal mucosa, due to the disturbance of the tongue papillae and taste buds. Hyperkeratosis and acanthosis also interfere with the display resolution of RCM (27). The features of RCM are difficult to identify in the deeper layer of the sections due to fact the upper tissue layer interferes with image formation (28). It has also been reported that in strongly keratinized lesions, images cannot be clearly identified (15). Subclinical inflammation and alteration may be present on the clinically unaffected areas which would also interfere with specificity values. Otherwise, it has been reported that it is difficult to distinguish between the range and type of infiltrating inflammatory cells (29), as well as between melanocytes and Langerhans cells (30). Furthermore, a more portable device would be helpful during manipulation (31). Recently a new video-mosaicking approach was reported for intraoral imaging, meaning this technique may be able to overcome the limitation of original confocal microscopy (32).

In conclusion, RCM may be a non-invasive, adjunctive tool that can be used to evaluate OLP in real-time and may be of use for the diagnosis of OLP to avoid unnecessary invasive procedures. Furthermore, RCM may potentially be used as an auxiliary tool to reduce the area of resection required when there are epithelial alterations that are not visible to the naked eye. The diagnostic value of RCM criteria needs to be further confirmed through a blinded evaluation comparing OLP and non-OLP lesions. Further research is necessary to determine the characteristics and use of RCM in OLP lesions for future clinical applications.

\section{Acknowledgements}

The authors would like to thank Dr Zhen Tian and Dr Lizhen Wang (Department of Oral Pathology, Ninth People's Hospital, Shanghai Jiao Tong University School of Medicine) for helping with pathological assessments.

\section{Funding}

The current study was supported by the National Nature Science Foundation of China (grant no. 81802694) and Seed Funding of Shanghai Ninth People's Hospital, Shanghai Jiao Tong University School of Medicine (grant no. JYZZ046).

\section{Availability of data and materials}

The datasets used and/or analyzed during the current study are available from the corresponding author on reasonable request.

\section{Authors' contributions}

YW and GZ designed the study. HP, GZ and LS collected the clinical data. HP and GZ performed the experiments and analyzed the data. HP and YWwrote the paper. All authors read and approved the final manuscript.

\section{Ethics approval and consent to participate}

Ethical approval for this investigation was obtained from the Ethics Committee of Shanghai Ninth People's Hospital, 
Shanghai Jiao Tong University School of Medicine (approval no. 2014038).

\section{Patient consent for publication}

Not applicable.

\section{Competing interests}

The authors declare that they have no competing interests.

\section{References}

1. Skrinjar I, Vidranski V, Brzak BL, Vidovic Juras D, Andabak Rogulj A, Brailo V and Vucicevic Boras V: Salivary cortisol levels in patients with oral lichen planus-A pilot case-control study. Dent J (Basel) 7: 59, 2019.

2. Lavanya N, Jayanthi P, Rao UK and Ranganathan K: Oral lichen planus: An update on pathogenesis and treatment. J Oral Maxillofac Pathol 15: 127-132, 2011.

3. Ismail SB, Kumar SK and Zain RB: Oral lichen planus and lichenoid reactions: Etiopathogenesis, diagnosis, management and malignant transformation. J Oral Sci 49: 89-106, 2007.

4. Jones KB and Jordan R: White lesions in the oral cavity: Clinical presentation, diagnosis, and treatment. Semin Cutan Med Surg 34 161-170, 2015.

5. Warnakulasuriya S, Reibel J, Bouquot J and Dabelsteen E: Oral epithelial dysplasia classification systems: Predictive value, utility, weaknesses and scope for improvement. J Oral Pathol Med 37: 127-133, 2008.

6. van der Waal I: Potentially malignant disorders of the oral and oropharyngeal mucosa; terminology, classification and present concepts of management. Oral Oncol 45: 317-323, 2009.

7. Mendes SF, de Oliveira Ramos G, Rivero ER, Modolo F, Grando LJ and Meurer MI: Techniques for precancerous lesion diagnosis. J Oncol 2011: 326094, 2011.

8. White WM, Rajadhyaksha M, González S, Fabian RL and Anderson RR: Noninvasive imaging of human oral mucosa in vivo by confocal reflectance microscopy. Laryngoscope 109: 1709-1717, 1999.

9. González S, Sánchez V, González-Rodríguez A, Parrado C and Ullrich M: Confocal microscopy patterns in nonmelanoma skin cancer and clinical applications. Actas Dermosifiliogr 105: 446-458, 2014

10. Maher NG, Collgros H, Uribe P, Ch'ng S, Rajadhyaksha M and Guitera P: In vivo confocal microscopy for the oral cavity: Current state of the field and future potential. Oral Oncol 54: 28-35, 2016.

11. Dental Supplement, Romano A, Santarelli A, Lajolo C, Della Vella F, Mascitti M, Serpico R and Contaldo M: Analysis of oral mucosa erosive-ulcerative lesions by reflectance confocal microscopy. J Biol Regul Homeost Agents 33 (Suppl 1): S11-S17, 2019.

12. Rajadhyaksha M, González S, Zavislan JM, Anderson RR and Webb RH: In vivo confocal scanning laser microscopy of human skin II: Advances in instrumentation and comparison with histology. J Invest Dermatol 113: 293-303, 1999.

13. Dental Supplement, Contaldo M, Lajolo C, Di Petrillo M, Ballini A, Inchingolo F, Serpico R and Romano A: Analysis of lip pigmentations by reflectance confocal microscopy: Report of two cases. J Biol Regul Homeost Agents 33 (Suppl 1): S19-S25, 2019.

14. Contaldo M, Di Stasio D, Petruzzi M, Serpico R and Lucchese A: In vivo reflectance confocal microscopy of oral lichen planus. Int J Dermatol 58: 940-945, 2019.

15. Contaldo M, Lauritano D, Carinci F, Romano A, Di Stasio D, Lajolo C, Della Vella F, Serpico R and Lucchese A: Intraoral confocal microscopy of suspicious oral lesions: A prospective case series. Int J Dermatol: Jul 9, 2019 (Epub ahead of print).
16. Alessi SS, Nico MM, Fernandes JD and Lourenco SV: Reflectance confocal microscopy as a new tool in the in vivo evaluation of desquamative gingivitis: Patterns in mucous membrane pemphigoid, pemphigus vulgaris and oral lichen planus. Br J Dermatol 168: 257-264, 2013.

17. Ardigo M,Donadio C,Franceschini C,Catricala C and Agozzino M: Interest of reflectance confocal microscopy for inflammatory oral mucosal diseases. J Eur Acad Dermatol Venereol 29: 1850-1853, 2015.

18. van der Meij EH and van der Waal I: Lack of clinicopathologic correlation in the diagnosis of oral lichen planus based on the presently available diagnostic criteria and suggestions for modifications. J Oral Pathol Med 32: 507-512, 2003.

19. Kurago ZB: Etiology and pathogenesis of oral lichen planus: An overview. Oral Surg Oral Med Oral Pathol Oral Radiol 122: 72-80, 2016.

20. Sugerman PB and Sabage NW: Oral lichen planus: Causes, diagnosis and management. Aust Dent J 47: 290-297, 2002.

21. Edwards PC and Kelsch R: Oral lichen planus: Clinical presentation and management. J Can Dent Assoc 68: 494-499, 2002.

22. Thongprasom K, Carrozzo M, Furness S and Lodi G: Interventions for treating oral lichen planus. Cochrane Database Syst Rev 7: CD001168, 2011.

23. Moscarella E, González S, Agozzino M, Sánchez-Mateos JL, Panetta C, Contaldo M and Ardigò M: Pilot study on reflectance confocal microscopy imaging of lichen planus: A real-time, non-invasive aid for clinical diagnosis. J Eur Acad Dermatol Venereol 26: 1258-1265, 2012.

24. Agozzino M,GonzálezS and Ardigò M:Reflectance confocal microscopy for inflammatory skin diseases. Actas Dermosifiliogr 107: 631-639, 2016 (In English, Spanish).

25. Irani S, Esfahani AM and Ghorbani A: Dysplastic change rate in cases of oral lichen planus: A retrospective study of 112 cases in an Iranian population. J Oral Maxillofac Pathol 20: 395-399, 2016.

26. Mattsson U, Jontell M and Holmstrup P: Oral lichen planus and malignant transformation: Is a recall of patients justified? Crit Rev Oral Biol Med 13: 390-396, 2002.

27. Incel P, Gurel MS and Erdemir AV: Vascular patterns of nonpigmented tumoral skin lesions: Confocal perspectives. Skin Res Technol 21: 333-339, 2015.

28. Contaldo M, Poh CF, Guillaud M, Lucchese A, Rullo R, Lam S, Serpico R, MacAulay CE and Lane PM: Oral mucosa optical biopsy by a novel handheld fluorescent confocal microscope specifically developed: Technologic improvements and future prospects. Oral Surg Oral Med Oral Pathol Oral Radiol 116: 752-758, 2013

29. Ardigò M, Maliszewski I, Cota C, Scope A, Sacerdoti G, Gonzalez S and Berardesca E: Preliminary evaluation of in vivo reflectance confocal microscopy features of discoid lupus erythematosus. Br J Dermatol 156: 1196-1203, 2007.

30. Hashemi P, Pulitzer MP, Scope A, Kovalyshyn I, Halpern AC and Marghoob AA: Langerhans cells and melanocytes share similar morphologic features under in vivo reflectance confocal microscopy: A challenge for melanoma diagnosis. J Am Acad Dermatol 66: 452-462, 2012.

31. Lucchese A, Gentile E, Romano A, Maio C, Laino L and Serpico R: The potential role of in vivo reflectance confocal microscopy for evaluating oral cavity lesions: A systematic review. J Oral Pathol Med 45: 723-729, 2016.

32. Peterson G, Zanoni DK, Ardigo M, Migliacci JC, Patel SG and Rajadhyaksha M: Feasibility of a video-mosaicking approach to extend the field-of-view for reflectance confocal microscopy in the oral cavity in vivo. Lasers Surg Med: May 8, 2019 (Epub ahead of print).

This work is licensed under a Creative Commons Attribution-NonCommercial-NoDerivatives 4.0 International (CC BY-NC-ND 4.0) License. 